\title{
Cross-over or within-participants design
}

\section{David F Marks}

Potential competing interests: The author(s) declared that no potential competing interests exist.

The cross-over or within-participants design is used when the same people provide measures of a dependent variable at more than one time and differences between the measures at the different times are recorded. 